\title{
EVEN GENERATORS IN THE Mod 2 COHOMOLOGY OF A FINITE H-SPACE
}

\author{
JAMES P. LIN*
}

\section{Introduction.}

The following conjecture has been attacked by many mathematicians:

Conjecture. If $X$ is a simply connected finite $\mathrm{H}$-space then $H_{*}(\Omega X ; \mathrm{Z})$ is two torsion free.

The author has shown $H_{*}(\Omega X ; \mathrm{Z})$ has no odd torsion [8]. The corresponding theorem for $X$, a Lie group was proven by Bott [2]. Recently, R. Kane proved the following theorems:

THEOREM A (Kane) [6]. $H_{*}(\Omega X ; Z)$ is two torsion free if and only if there are no even generators in $H^{*}\left(X ; Z_{2}\right)$.

THEOREM B (Kane) [5]. There are no even generators of $H^{*}\left(X ; Z_{2}\right)$ of degree congruent to zero mod four.

Progress has been slow in eliminating generators of degree congruent to two mod four. One of the difficulties is the existence of odd degree elements of $H^{*}\left(X ; Z_{2}\right)$ whose cup product square is nonzero. This feature of the cohomology does not occur for odd primes. Another difficulty is that Theorem $\mathrm{A}$ is not true for non-simply connected $\mathrm{H}$-spaces $X$. This is shown in the cohomology of the Lie group $\operatorname{Ad} E_{7}$ :

$$
H^{*}\left(\operatorname{Ad} E_{7} ; Z_{2}\right) \cong Z_{2}\left[x_{1}, x_{5}, x_{9}\right] /\left(x_{1}^{4}, x_{5}^{4}, x_{9}^{4}\right) \otimes \Lambda\left(x_{6}, x_{15}, x_{17}, x_{23}, x_{27}\right)
$$

which has a six dimensional generator.

The purpose of this paper is to study the module of even indecomposables $Q H^{\text {even }}\left(X ; Z_{2}\right)$ as a module over the Steenrod algebra. Throughout the paper we will assume $H_{*}\left(X ; Z_{2}\right)$ is associative. It will soon become evident that this

* Author was partially supported by the National Science Foundation and the Sloan Foundation.

Received October 30, 1978. 
assumption considerably simplifies the coalgebra structure of $H^{*}\left(X ; \mathrm{Z}_{2}\right)$. Let $a(2)^{+}$be the subalgebra of $a(2)$ generated by elements of the form $\mathrm{Sq}^{2 l}$ for $l \geqq 0$.

We will prove that as a module over $G(2)^{+}, Q H^{\text {even }}\left(X ; Z_{2}\right)$ is generated by elements of degree $2^{n}-2$ for $n>1$. Furthermore, we will exhibit explicit Steenrod squares that connect all even generators to generators of degree $2^{n}-2$ for $n>1$. Theorem A therefore implies, if $\sum_{n=2}^{\infty} Q H^{2^{n}-2}\left(X ; Z_{2}\right)$ is trivial, then $H_{*}(\Omega X ; \mathrm{Z})$ is two torsion free.

The contents of this paper has been partly joint work with John Harper. Much of the flavor is taken from a previous incorrect version, which showed us that the $Z_{2}$ cohomology of a finite $H$-space is really an order of magnitude more complicated than the $\bmod p$ cohomology. The techniques used in this paper rely heavily on ideas of Zabrodsky. It might be said that Zabrodsky's ideas have pervaded everyone's work on the theory of finite $H$-spaces in the last ten years. This paper is no exception. Finally, I would like to thank David Kraines for several helpful discussions.

\section{Hopf algebras over the Steenrod algebra.}

To begin our study we first need to gather information about the coproducts of generators of the cohomology of a finite $H$-space $X$. We assume throughout that $X$ is a finite $H$-space with associative $\bmod 2$ homology ring.

There are two theorems already proven that will be used:

THeOREM 1.1 (Browder) [3]. If $t$ and $\dot{u}$ are odd homology primitives of $H_{*}\left(X ; Z_{2}\right)$ then

(1) $t^{2}=0$

(2) $[t, u]=0$.

Proof. By Browder [3] there are no even integral primitives in $H_{*}\left(X ; Z_{2}\right)$. This implies $t \beta^{i}=u \beta^{i}=0$ for all $i$. By induction using the fact that $\beta^{i}$ is a derivation, we get $\left(t^{2}\right) \beta^{i}=[t, u] \beta^{i}=0$. Hence $t^{2}$ and $[t, u]$ are even integral primitives. It follows that they both vanish.

Corollary 1.2. If $\Lambda \subset H_{*}\left(X ; Z_{2}\right)$ is the subHopf algebra generated by $\mathrm{PH}_{\mathrm{odd}}\left(\mathrm{X} ; \mathrm{Z}_{2}\right)$ then $\Lambda$ is an exterior algebra over the Steenrod algebra.

Proof. By Browder $\Lambda$ is an exterior algebra and for $t \in P(\Lambda), t \beta^{1}=0$.

THEOREM 1.3 (Kane) [5]. There exists a subHopf algebra $B \cong H^{*}\left(X ; Z_{2}\right)$ over the Steenrod algebra with the following properties 
(1) $B$ is concentrated in even degrees

(2) The induced map

$$
Q B^{\text {even }} \rightarrow Q H^{\text {even }}\left(X ; \mathrm{Z}_{2}\right)
$$

is an epimorphism.

Proof. Let $\Lambda$ be the homology Hopf algebra described in Corollary 1.2. We will show $\Lambda$ is normal in $H_{*}\left(X ; Z_{2}\right)$. Let $\Lambda(n)$ be the subHopf algebra of $\Lambda$ generated by primitives of degree greater than or equal to $n$. Because $H_{*}\left(X ; Z_{2}\right)$ is finite dimensional, $\Lambda(n)$ is finite, and vanishes for large $n$.

Suppose the highest odd primitive occurs in degree $m$. Then $\Lambda(m)$ is normal in $H_{*}\left(X ; Z_{2}\right)$. To see this, it suffices to show $\Lambda(m)$ is central in $H_{*}\left(X ; Z_{2}\right)$. Because $H_{*}\left(X ; Z_{2}\right)$ is associative, we can test the centrality on generators of $H_{*}\left(X ; \mathrm{Z}_{2}\right)$. Let $t \in \Lambda(m)$ be a generator and suppose that $t$ commutes with all generators of degree less than $l$. Then if $u$ is a generator of degree $l,[t, u]$ has coproduct

$$
\begin{aligned}
\Delta[t, u]= & {[t, u] \otimes 1+1 \otimes[t, u]+\sum\left[t, u_{i}^{\prime}\right] \otimes u_{i}^{\prime \prime}+} \\
& +\sum u_{i}^{\prime} \otimes\left[t, u_{i}^{\prime \prime}\right] \quad \text { if } \bar{\Delta} u=\sum u_{i}^{\prime} \otimes u_{i}^{\prime \prime},
\end{aligned}
$$

but by induction $\left[t, u_{i}^{\prime}\right]=0=\left[t, u_{i}^{\prime \prime}\right]$.

Therefore $[t, u]$ is primitive. Now if $u$ is of even degree $[t, u]$ has degree greater than $m$. Hence by construction $[t, u]=0$. If $u$ has odd degree it may be chosen to be primitive because the map

$$
P H_{\text {odd }}\left(X ; \mathrm{Z}_{2}\right) \rightarrow Q H_{\text {odd }}\left(X ; \mathrm{Z}_{2}\right)
$$

is an epimorphism. Therefore by Corollary $1.2,[t, u]=0$. Hence $\Lambda(m)$ is central in $H_{*}\left(X ; Z_{2}\right)$.

Assume by induction that $\Lambda(n+1)$ is normal in $H_{*}\left(X ; \mathbf{Z}_{2}\right)$. We will show that $\Lambda(n)$ is normal. We have $\Lambda(n) / / \Lambda(n+1)$ is a subHopf algebra of $H_{*}\left(X ; Z_{2}\right) / / \Lambda(n+1)$ and is exterior on generators of degree $n$. Furthermore the highest odd degree primitives of $H_{*}\left(X ; Z_{2}\right) / / \Lambda(n+1)$ occur in degree $n$. By exactly the same argument as above, $\Lambda(n) / / \Lambda(n+1)$ is central in $H_{*}\left(X ; \mathrm{Z}_{2}\right) / / \Lambda(n+1)$.

It follows that $\Lambda(n)$ is normal in $H_{*}\left(X ; Z_{2}\right)$. By induction it follows that $\Lambda(0)$ $=\Lambda$ is normal in $H_{*}\left(X ; Z_{2}\right)$. If we define $B=\left(H_{*}\left(X ; Z_{2}\right) / / \Lambda\right)^{*}$ then $B$ satisfies the requirements of the theorem.

Corollary 1.4. $H^{*}\left(X ; Z_{2}\right) / / B$ is a primitively generated exterior algebra.

Proof. We have $\Lambda^{*}=H^{*}\left(X ; Z_{2}\right) / / B$. Consider $\xi \Lambda^{*}$. If $\xi \Lambda^{*}$ is nontrivial then 
$P\left(\xi \Lambda^{*}\right)$ is nontrivial. Dually, there would be an even generator in $\Lambda$. This is impossible. Then $\Lambda^{*}$ is exterior and by the Samelson Leray theorem, $\Lambda^{*}$ is primitively generated.

We can now prove an important theorem about the coproduct of generators of $H^{*}\left(X ; Z_{2}\right)$ :

THEOREM 1.5. There is a choice of representatives for $Q H^{\text {odd }}\left(X ; Z_{2}\right)$ with the property that they have reduced coproduct in $B \otimes H^{*}\left(X ; Z_{2}\right)$.

Proof. Let $\pi: A \rightarrow C$ be an epimorphism of connected Hopf algebras and let $\pi: C \rightarrow I(C)$ be the projection onto the augmentation ideal. We may define a map $g: A \rightarrow A \otimes I(C)$ by

$$
g: A \rightarrow A \otimes A \stackrel{1 \otimes \pi}{\longrightarrow} A \otimes C \stackrel{1 \otimes \ell}{\longrightarrow} A \otimes I(C) .
$$

Let $T=$ kernel $g$. Baum and Browder [1] prove the following:

(1.1) If $C$ is primitively generated and $T$ is a Hopf algebra then there is a module $R \subset A$ with the property that the sequence

$$
0 \rightarrow T \rightarrow R \rightarrow P(C) \rightarrow 0
$$

is exact and $\bar{\Delta}(I(R)) \subset I(T) \otimes I(R)$.

To apply this result to $C=H^{*}\left(X ; Z_{2}\right) / / B$ we have to check that $T$ is a Hopf algebra. Consider the dual of $g$.

$$
\begin{gathered}
g_{*}: H_{*}\left(X ; \mathrm{Z}_{2}\right) \otimes I(\Lambda) \rightarrow H_{*}\left(X ; \mathrm{Z}_{2}\right) \otimes \Lambda \rightarrow H_{*}\left(X ; \mathrm{Z}_{2}\right) \otimes H_{*}\left(X ; \mathrm{Z}_{2}\right) \\
\rightarrow H_{*}\left(X ; \mathrm{Z}_{2}\right) .
\end{gathered}
$$

We have image $g_{*}=H_{*}\left(X ; Z_{2}\right) I(\Lambda)$. Hence. $T=$ cokernel $g_{*}$ is $B$. Hence (1.1) applies. There is an exact sequence

$$
0 \rightarrow B \rightarrow R \rightarrow P\left(H^{*}\left(X ; Z_{2}\right) / / B\right) \rightarrow 0,
$$

with $\bar{L}(I(R)) \subseteq I B \otimes I(R), R \cong H^{*}\left(X ; \mathrm{Z}_{2}\right)$. Now since there is an extension

$$
\mathrm{Z}_{2} \rightarrow B \rightarrow H^{*}\left(X ; \mathrm{Z}_{2}\right) \rightarrow H^{*}\left(X ; \mathrm{Z}_{2}\right) / / B \rightarrow \mathrm{Z}_{2}
$$

there is an isomorphism

$$
Q H^{\text {odd }}\left(X ; \mathrm{Z}_{2}\right) \cong Q\left(H^{*}\left(X ; \mathrm{Z}_{2}\right) / / B\right)^{\text {odd }} \cong P\left(H^{*}\left(X ; \mathrm{Z}_{2}\right) / / B\right)^{\text {odd }}
$$

by 1.3 and 1.4 . This completes the proof.

Proposition 1.6. Suppose an element $x \in A$ is decomposable and has reduced 
coproduct in $B \otimes B$ where $B$ is a subHopf algebra that contains $\xi A$. Then we may write $x=y^{2}+d$ where $d \in B$ and $d$ is decomposable in $B$.

Proof. Consider $\{x\} \in A / / B .\{x\}$ is decomposable primitive hence $\{x\}$ $=\{y\}^{2}$. Therefore $x-y^{2} \in I(B) A$ and since $\xi A \cong B, \bar{\Delta}\left(x-y^{2}\right) \in B \otimes B$. The Milnor-Moore isomorphism $A \cong B \otimes A / / B$ is the map

$$
\theta: A \stackrel{\Delta}{\longrightarrow} A \otimes A \stackrel{g \otimes \pi}{\longrightarrow} B \otimes A / / B
$$

where $g$ is a $B$-module splitting. Under this isomorphism $B$ is identified with $B \otimes 1$. Applying $\theta\left(x-y^{2}\right)=\left(x-y^{2}\right) \otimes 1$. Hence $x-y^{2} \in B$.

Nuw consider the subHopf algebra of $B$ generated by all elements of degree less than or equal to half the degree of $x-y^{2}$. Call this Hopf algebra $B_{1}$. Then the residue class $\left\{x-y^{2}\right\}$ in $A / / B_{1}$ is primitive decomposable. Hence $\left\{x-y^{2}\right\}$ $=\{z\}^{2}$. So $\left\{x-(y+z)^{2}\right\}=0$ in $A / / B_{1}$. Consider the induced map

$$
\begin{gathered}
0 \rightarrow P\left(B / / B_{1}\right) \rightarrow P\left(A / / B_{1}\right) \\
\left\{x-(y+z)^{2}\right\} \rightarrow 0 .
\end{gathered}
$$

It follows that $x-(y+z)^{2} \in I\left(B_{1}\right) B$. Now since $Q B_{1}$ is bounded by half the degree of $x-(y+z)^{2}$ it follows that $x-(y+z)^{2}$ is decomposable in $B$.

\section{Secondary operations.}

In this section, we will describe a secondary operation that detects the square of a homology class. The ideas are described in detail in [8]. The following theorem is a slight variation of the theorem described in [8].

THEOREM 2.1. Let $x$ be a $2 n$ dimensional cohomology generator with coproduct in $C \otimes C$ where $C$ is an $a(2)$ subHopf algebra of $H^{*}\left(X ; Z_{2}\right)$. Suppose $x=\operatorname{Sq}^{1} y$ where $\bar{\Delta} y \in C \otimes C, y \notin C$. Furthermore suppose $\mathrm{Sq}^{2 n+1} \mathrm{Sq}^{1}=\sum a_{i} b_{i}$ and $b_{i} y$ is decomposable in $C$. Then there is a secondary operation $\varphi$ defined on $x$ and

$$
\left.\bar{\Delta} \varphi(x)=x \otimes x+\sum \operatorname{im} a_{i}+I(C) H^{*} \otimes H^{*}+H^{*} \otimes I(C)\right) H^{*} .
$$

If $t \in P H_{2 n}\left(X ; Z_{2}\right)$ satisfies $\langle t, x\rangle \neq 0,\left\langle t \otimes t, \sum \operatorname{im} a_{i}\right\rangle=0$, and $\langle t, C\rangle=0$ then $t^{2}$ $\neq 0$.

Proof. The proof is exactly the same as Main Theorem 1.3 of [8] with the exception that the $K$ in the Toda Bracket diagram becomes $K\left(Z_{2}, 2 n-1\right)$ and represents $y$.

The following theorem is due to Kane [5]: 
THEOREM 2.2 (Kane). Let $X$ be a finite $H$-space. Then $Q H^{4 k}\left(X ; Z_{2}\right)=0$ for all $k \geqq 1$.

Now let $a(2)^{+}$be the subalgebra of $a(2)$ generated by elements of the form $\mathrm{Sq}^{2 n}$ for $n=1,2, \ldots$ Let $Q^{\text {even }}$ be the submodule of $Q H\left(X ; \mathrm{Z}_{2}\right)$ concentrated in even degrees. Then $Q^{\text {even }}$ admits an $a(2)^{+}$action. Our next theorem describes the degrees of the $G(2)^{+}$module generators of $Q^{\text {even }}$.

THEOREM 2.3. $Z_{2} \otimes_{I\left(G(2)^{+}\right)} Q^{\text {even }}$ is concentrated in degrees of the form $2^{n}-2$ for $n \geqq 2$.

Proof. Define inductively $Q_{0}=\mathrm{Sq}^{1}, Q_{1}=\left[\mathrm{Sq}^{1}, \mathrm{Sq}^{2}\right], \ldots Q_{n}=\left[Q_{n-1}, \mathrm{Sq}^{2^{n}}\right]$.

Applying formulas in the Milnor basis [9], we obtain

(2.1.1) The $Q_{i}$ 's generate a primitively generated exterior subHopf algebra of $a(2)$.

$$
Q_{i} \mathrm{Sq}^{l}=\mathrm{Sq}^{l} Q_{i}+Q_{i+1} \mathrm{Sq}^{l-2^{i+1}}
$$

where the last term is omitted if $l<2^{i+1}$.

Let $v(s)=2^{s+1}-1$. Then applying (2.1.2)

$$
\mathrm{Sq}^{2 n+1}=\sum_{s=-1} \mathrm{Sq}^{2 n-2 v(s)} Q_{s+1}
$$

(2.1.3) implies

(2.1.4) If $2 n+1 \neq v(s)$ for any $s$, then $\mathrm{Sq}^{2 n+1}$ belongs to the right ideal generated by $a(2)^{+}$and the left ideal generated by the $Q_{i}$ 's.

Returning to the theorem, suppose $\bar{x} \in Q^{\text {even }}$ has nonzero projection in $Z_{2} \otimes_{I\left(a(2)^{+}\right)} Q^{\text {even }}$ and is not of degree $2^{n}-2=2 v(n-1)$. We will show this leads to a contradiction. Let degree $\bar{x}=2 l$. By Theorem 2.2, $l$ is odd. By Theorem 1.3 $\bar{x}$ has a representative $x \in B$ where $B$ is an even $a(2)$ subHopf algebra of $H^{*}\left(X ; Z_{2}\right)$.

Filter $B$ by $B(s) \subseteq B$ where $B(s)$ is the $G(2)$ subHopf algebra of $B$ generated by elements of degree less than or equal to $s$. Suppose $x \in B(m+1), x \notin B(m)$. Then $\bar{\Delta} x \in B(m) \otimes B(m)$ and $Q_{i} x=0$. Now $2 l+1 \neq v(s)$ so

$$
\mathrm{Sq}^{2 l+1}=\sum \mathrm{Sq}^{2 l-2 v(s)} Q_{s+1}
$$

where $2 l-2 v(s) \neq 0$ for all $s$.

Applying the Main Theorem 1.3 of [8] we get a class $\varphi(x) \subset H^{4 l}\left(X ; Z_{2}\right)$ with

$$
\begin{aligned}
\bar{\Delta} \varphi(x)=x \otimes x+\sum i m \mathrm{Sq}^{2 l-2 v(s)} & +I B(m) H^{*} \otimes H^{*}+ \\
& +H^{*} \otimes I B(m) H^{*} .
\end{aligned}
$$


Let $t \in P H_{2 l}\left(X ; \mathrm{Z}_{2}\right)$ satisfy $\langle t, x\rangle \neq 0,\langle t, B(m)\rangle=0$. Then if $\left\langle t \otimes t, \mathrm{im} \mathrm{Sq}^{2 l-2 v(s)}\right\rangle$ $=0$ for all $s,(2.1 .5)$ would imply

$$
\left\langle t^{2}, \varphi(x)\right\rangle \neq 0 .
$$

This would imply $Q^{4 l} \neq 0$ which contradicts Theorem 2.2 . Therefore

$$
\left\langle t \otimes t, \mathrm{im} \mathrm{Sq}^{2 l-2 v(s)}\right\rangle \neq 0 \quad \text { for some } s .
$$

Now since $\bar{x}$ has nonzero projection in $\mathrm{Z}_{2} \otimes_{I\left(i(2)^{+}\right)} Q^{\text {even }}$ we may assume $\left\langle t, \operatorname{im} G(2)^{+}\right\rangle=0$. Therefore the only possible way $\left\langle t \otimes t, \mathrm{im} \mathrm{Sq}^{2 l-2 v(s)}\right\rangle \neq 0$ for some $s$ is if $x=\mathrm{Sq}^{1} y$ for some $y$.

We conclude $x=\mathrm{Sq}^{1} y$ for some $y$. Now consider $\bar{x}$ in $Q H^{*}\left(X ; \mathrm{Z}_{2}\right)$. We have just shown that $\bar{x}$ is in the image of Steenrod operations of odd degree. By (2.1.3) and (2.1.4), the only odd degree Steenrod operations that can hit $\bar{x}$ are of degree $v(i)$ for some $i$ and we may write

$$
\bar{x}=\sum_{i=1}^{l} \mathrm{Sq}^{v(i)} \bar{z}_{i}=\sum_{i=1}^{l} Q_{i} \bar{z}_{i}
$$

where we may assume

$$
\bar{z}_{i} \text { and } \mathrm{Sq}^{v(i)} \bar{z}_{i} \text { are not in the image of } a(2)^{+} \text {. }
$$

Consider the largest of all terms appearing in (2.1.6)

$$
\mathrm{Sq}^{v(l)} \bar{z}_{l} \text {. }
$$

According to Theorem 1.5 we may assume $\bar{z}_{l}$ has representative $z_{l}$ with $\bar{\Delta} z_{l} \in B \otimes H^{*}\left(X ; Z_{2}\right)$. Pick representatives $w_{1}, \ldots, w_{3}$ for all odd generators of degree less than degree of $z_{l}$ with the property $\bar{\Delta} w_{i} \in B \otimes H^{*}\left(X ; Z_{2}\right)$. Define $C$ to be the $a(2)$ subHopf algebra generated by $B(2 l-1)$ and $w_{1}, \ldots, w_{s}$. Finally, let $y=\mathrm{Sq}^{2 v(l-1)} z_{l}$ so that $\mathrm{Sq}^{1} \cdot y=\mathrm{Sq}^{v(l)} z_{l}$. Then

$$
\bar{\Delta} y \in C \otimes C \quad \text { and } \quad y \notin C .
$$

The first statement follows by construction. If $y \in C$ that would imply $\mathrm{Sq}^{v(l)} \bar{z}_{l}$ is in the image of $a(2)^{+}$which contradicts (2.1.7). We now apply Theorem 2.1. By assumption, $2 l=$ degree $\mathrm{Sq}^{1} y \neq 2^{n}-2$ hence

$$
\mathrm{Sq}^{2 l+1}=\sum_{r=-1} \mathrm{Sq}^{2 l-2 v(r)} Q_{r+1} \quad \text { where } 2 l>2 v(r) \text { for all } r .
$$

Hence

$$
\mathrm{Sq}^{2 l+1} \mathrm{Sq}^{1}=\sum_{r=0} \mathrm{Sq}^{2 l-2 v(r)} \mathrm{Sq}^{1} Q_{r+1}
$$

Here we used $\mathrm{Sq}^{1} \mathrm{Sq}^{1}=0$ and $\mathrm{Sq}^{1} Q_{r+1}=Q_{r+1} \mathrm{Sq}^{1}$. Now, by Theorem 2.2 
(2.19) $Q_{r+1} y$ is decomposable of degree $\equiv 0 \bmod 4$ and $\bar{\Delta} Q_{r+1} y \in C \otimes C$.

Proposition 1.6 implies $Q_{r+1} y=z^{2}+d$ where $d$ is decomposable in $C$. Now if $z$ is decomposable then $z^{2}$ is decomposable in $C$ because $C$ contains $\xi H^{*}\left(X ; \mathrm{Z}_{2}\right)$ up to dimension $2 l-2$. If $z$ is indecomposable, we may assume $z=z_{1}+d_{1}$ where $z_{1} \in B$ by Theorem 1.3. Hence $z^{2}$ is always decomposable in $C$ and hence $Q_{r+1} y$ is decomposable in $C$.

Now since $\mathrm{Sq}^{1} y$ is not in the image of $a(2)^{+}$(by 2.1.7), there is a $t \in P H_{2 l}\left(X ; Z_{2}\right)$ with

$$
\left\langle t, \mathrm{Sq}^{1} y\right\rangle \neq 0 \text { and }\left\langle t, C+\operatorname{im} a(2)^{+}\right\rangle=0 .
$$

It follows that

(2.1.11) $\left\langle t \otimes t, \mathrm{Sq}^{2 l-2 v(r)} \mathrm{Sq}^{1}\right\rangle=0$ by the Cartan Formula and the result that $t$ is annihilated by any Steenrod operation involving two $\mathrm{Sq}^{1} \mathrm{~s}$ (by Browder [3]).

By Theorem $2.1 t^{2} \neq 0$. This implies $Q^{4 l} \neq 0$ which contradicts Theorem $2.2 \mathrm{We}$ conclude that $\mathrm{Sq}^{v(l)} \bar{z}_{l}$ must be in the image of $a(2)^{+}$which implies $\bar{x}$ is in the image of $a(2)^{+}$. This completes the proof.

\section{Action of $a(2)^{+}$on $Q^{8 k+2}$.}

In Section 2 it was shown that elements of degree not equal to $2^{n}-2$ for some $n$ belong in the image of Steenrod operations $a(2)^{+}$. One drawback to this result is that we do not know which Steenrod operations connect the even generators. In this section, we use Main Theorem 1.3 of [8] to specify certain Steenrod algebra connections.

Our first task will be to describe the even subHopf algebra $B$ in more precise terms. Recall that $B \cong H^{*}\left(X ; \mathbf{Z}_{2}\right)$ satisfies $Q B^{\text {even }} \rightarrow Q H^{\text {even }}\left(X ; Z_{2}\right)$ epimorphic and $B$ is concentrated in even degrees.

Proposition 3.1. $B / / \xi B$ is an exterior algebra on generators of degree congruent to two mod four.

Proof. Let $x$ be a generator of $B / / \xi B$ of degree $2 l$. Let $B_{1}$ be the subHopf algebra of $B / / \xi B$ generated by elements of degree less than $2 l$. Then we have an exact sequence

$$
0 \rightarrow P(B / / \xi B) / / B_{1} \stackrel{\theta}{\rightarrow} P\left(H^{*} / / \xi B\right) / / B_{1} .
$$

Now if degree $x \equiv 0 \bmod 4$, then by Theorem $2.2 \theta\{x\}=0$ so $\{x\}=0$ and $x$ belongs to the ideal generated by $B_{1}$. But then $x$ could not be a generator of 
$B / / \xi B$. We conclude that all generators of $B / / \xi B$ are concentrated in degrees congruent to two mod 4.

Proposition 3.2. $B / / \xi B$ is primitively generated.

Proof. It suffices to show by Milnor-Moore [10] that $(B / / \xi B)_{*}$ is commutative, associative and has no squares. By hypothesis $(B / / \xi B)_{*}$ is associative. Hence we may test commutativity on generators. Given the lowest degree commutator say $[t, u]$, we may assume $t, u$ are algebra generators. Hence they are dual to primitives. The only primitives of $B / / \xi B$ occur in degrees congruent to $2 \bmod 4$. Therefore degree $[t, u]$ is congruent to zero mod 4. This contradicts the fact that $Q(B / / \xi B)$ is concentrated in degrees congruent to zero $\bmod 4$. Hence $(B / / \xi B)_{*}$ is commutative and associative. Therefore the squaring map is a map of Hopf algebras. Once again, if there are squares, there must be primitive squares of generators. But all such squares occur in degrees congruent to zero $\bmod 4$. Hence $B / / \xi B$ is primitively generated.

We now apply the technique of Baum and Browder [1] to select representatives for $Q B$ :

THEOREM 3.3. There is a choice of representatives for $Q B$ with reduced coproduct in $\xi B \otimes B$.

Proof. There is an extension of Hopf algebras

$$
\mathrm{Z}_{2} \rightarrow \xi B \rightarrow B \rightarrow B / / \xi B \rightarrow \mathrm{Z}_{2} .
$$

By Proposition $3.2 B / / \xi B$ is primitively generated. Consider the map

$$
g: B \rightarrow B \otimes B \stackrel{1 \otimes \pi}{\longrightarrow} B \otimes B / / \xi B \rightarrow B \otimes I(B / / \xi B) .
$$

We have kernel $g=\xi B$.

Following equation (1.1) we have a choice of representatives for $Q B$ $\cong Q(B / / \xi B)$ that have the desired coproduct.

Proposition 3.4. $Q^{4 l} B=0$ for all $l \geqq 1$.

Proof. This follows from Proposition 3.1 and

$$
Q B \cong Q(B / / \xi B) .
$$

Lemma 3.5. Suppose $x, y$ belong to $B$ have $\bar{\Delta} x \in \xi B \otimes B, \bar{\Delta} y \in \xi B \otimes B$, degree $x$ $=$ degree $y \equiv 2 \bmod 4$ and $x-y$ is decomposable in $B$. Then $x-y=0$. 
Proof. $\{x-y\} \in B / / \xi B$ is decomposable primitive. Since degree $x-y \equiv 2$ $\bmod 4,\{x-y\}=0$. Therefore $x-y \in I(\xi B) B$.

Applying the isomorphism $B \cong \xi B \otimes B / / \xi B$ shows $x-y \in \xi B$. But $\xi B$ is concentrated in degrees congruent to zero $\bmod 4$. Hence $x-y=0$.

We now prove a theorem about generators of $Q B$. We consider this theorem as a starting point for more general theorems which will be proved later. It is important to take note of the techniques used in the proof.

THEOREM 3.6. For all $k \geqq 1$

$$
Q B^{8 k+2}=\mathrm{Sq}^{4} Q B^{8 k-2}+\mathrm{Sq}^{4 k} Q B^{4 k+2}
$$

and

$$
\mathrm{Sq}^{4} Q B^{8 k+2}=0 \text {. }
$$

Furthermore if $\bar{x} \in Q B^{8 k+2}$ has representative $x \in B^{8 k+2}$ with $\bar{\Delta} x \in \xi B \otimes B$ and $x$ indecomposable in $H^{8 k+2}\left(X ; \mathrm{Z}_{2}\right)$, then $x=\mathrm{Sq}^{4} y+\mathrm{Sq}^{4 k} z$ for some $y, z$ with reduced coproduct in $\xi B \otimes B$ and $\mathrm{Sq}^{4} x=0$.

Proof. Note that it is possible that $Q B^{8 k+2}$ is not isomorphic to $Q H^{8 k+2}\left(X ; \mathrm{Z}_{2}\right)$. In fact if $x \in B^{8 k+2}, x=w^{2}$ where $w$ is a generator, then $\bar{x} \in Q B^{8 k+2}$. In this case we may choose $w$ with coproduct $\bar{\Delta} w \in B \otimes H^{*}\left(X ; Z_{2}\right)$. Then

$$
w^{2}=\mathrm{Sq}^{4 k+1} w=\mathrm{Sq}^{4 k} \mathrm{Sq}^{1} w+\mathrm{Sq}^{2} \mathrm{Sq}^{1} \mathrm{Sq}^{4 k-2} w .
$$

$\mathrm{Sq}^{1} \mathrm{Sq}^{4 k-2} w$ is decomposable in $B$. Hence $\bar{x}=\mathrm{Sq}^{4 k} \overline{\mathrm{Sq}^{1} w}$ in $Q B$.

Now consider the case when $k=1,8 k+2=10$.

Theorem 2.3 implies $Q H^{10}\left(X ; Z_{2}\right)$ is in the image of $a(2)^{+}$. By the above, $\overline{w_{5}^{2}}$ $=\mathrm{Sq}^{4} \bar{y}_{6}$ in $Q B$. We have $Q H^{10}\left(X ; \mathrm{Z}_{2}\right)$ must come from $Q H^{6}\left(X ; \mathrm{Z}_{2}\right)$ by Steenrod operations. Because $\mathrm{Sq}^{2} \mathrm{Sq}^{2}=\mathrm{Sq}^{3} \mathrm{Sq}^{1}$, the only possible operation is $\mathrm{Sq}^{4}$. Therefore

(3.1.1) $Q B^{10}=\mathrm{Sq}^{4} Q B^{6}, \quad$ and $\quad \mathrm{Sq}^{4} Q B^{10}=\mathrm{Sq}^{4} \mathrm{Sq}^{4} Q B^{6}=0$. Hence if $\bar{\Delta} x \in \xi B \otimes B, \bar{\Delta} y \in \xi B \otimes B$, and $x=\mathrm{Sq}^{4} y$ mod decomposables, then by Lemma $3.5 x=\mathrm{Sq}^{4} y$ and $\mathrm{Sq}^{4} x=0$.

Henceforth we will assume $k>1$, and $x \neq w^{2}$.

Now $B$ is a finite Hopf algebra, so we may apply downward induction. Assume that the theorem is true for all $Q B^{8 k^{\prime}+2}$ where $k^{\prime}>k$, and let $\bar{x} \in Q B^{8 k+2}$. Pick a representative $x \in B$ for $\bar{x}$ with $\bar{\Delta} x \in \xi B \otimes B$. Since $k>1$ there is a factorisation 


$$
\mathrm{Sq}^{1} \mathrm{Sq}^{8 k+2}=\mathrm{Sq}^{8 k+2} \mathrm{Sq}^{1}+\mathrm{Sq}^{8 k} Q_{1}+\left(Q_{2}+\mathrm{Sq}^{v(2)}\right) \mathrm{Sq}^{8 k-4} \text {. }
$$

I claim $\mathrm{Sq}^{8 k-4} x=0$. To see this, by the Adem relations

$$
\mathrm{Sq}^{8 k-4}=\mathrm{Sq}^{4} \mathrm{Sq}^{8 k-8}+\mathrm{Sq}^{8 k-5} \mathrm{Sq}^{1}+\mathrm{Sq}^{8 k-6} \mathrm{Sq}^{2} \text {. }
$$

Now $\mathrm{Sq}^{1} x=0$ and $\mathrm{Sq}^{2} x \in \xi B$ by Proposition 3.4 and Lemma 3.5. Hence $\mathrm{Sq}^{8 k-6} \mathrm{Sq}^{2} x=0$. It remains to show $\mathrm{Sq}^{4} \mathrm{Sq}^{8 k-8} x=0$. By induction, and Lemma 3.5, either $\mathrm{Sq}^{8 k-8} x=0$ or $\mathrm{Sq}^{8 k-8} x=\mathrm{Sq}^{4} y+\mathrm{Sq}^{8 k-4} z$ mod decomposables in $B$. But if $\bar{\Delta} y$ and $\bar{\Delta} z$ belong to $\xi B \otimes B$ then

$$
\mathrm{Sq}^{8 k-8} x=\mathrm{Sq}^{4} y+\mathrm{Sq}^{8 k-4} z \text {. }
$$

Hence by the Adem relations

$$
\mathrm{Sq}^{4} \mathrm{Sq}^{8 k-8} x=\mathrm{Sq}^{6} \mathrm{Sq}^{2} y+\mathrm{Sq}^{7} \mathrm{Sq}^{1} y+\mathrm{Sq}^{8 k-2} \mathrm{Sq}^{2} z+\mathrm{Sq}^{8 k-1} \mathrm{Sq}^{1} z=0
$$

by Lemma 3.5. This shows $\mathrm{Sq}^{8 k-4} x=0$.

We now show $\bar{x} \in \mathrm{im} \mathrm{Sq}{ }^{4}+\mathrm{im} \mathrm{Sq}^{4 k}$. If not, we can assume $x \in B(m+1)$, and

$$
x \notin B(m)+\mathrm{im} \mathrm{Sq}^{4}+\mathrm{im} \mathrm{Sq}^{4 k}
$$

where $B(m) \subset B$ is the $a(2)$ subHopf algebra generated by elements of degree less than or equal to $m$. Therefore there is a primitive $t \in B_{*}$ with $\langle t, x\rangle \neq 0$ and $\left\langle t, B(m)+\mathrm{im} \mathrm{Sq}^{4}+\mathrm{im} \mathrm{Sq}{ }^{4 k}\right\rangle=0$.

Also, since $P B_{4 l}=0$ we have $\left\langle t, \mathrm{Sq}^{2}\right\rangle=0$. I claim

$$
\left\langle t \otimes t, \mathrm{im} \mathrm{Sq}^{8 k+2}+\mathrm{im} \mathrm{Sq}^{8 k}+\operatorname{im} Q_{2}+\mathrm{Sq}^{v(2)}\right\rangle=0 .
$$

If this is true, Main Theorem 1.3 of [8] would imply $0 \neq t^{2} \in P B_{16 k+4}$ which is a contradiction. We proceed:

If $\left\langle t \otimes t, \mathrm{Sq}^{8 k+2}\right\rangle \neq 0 \Rightarrow x=w^{2}$ which is not possible.

If $\left\langle t \otimes t, \mathrm{im} \mathrm{Sq}^{8 k}\right\rangle \neq 0$ that would imply $x=\mathrm{Sq}^{4 k} y$.

If $\left\langle t \otimes t, \operatorname{im} Q_{2}+\mathrm{Sq}^{v(2)}\right\rangle \neq 0$, then

$$
Q_{2}+\mathrm{Sq}^{v(2)}=\mathrm{Sq}^{6} \mathrm{Sq}^{1}+\mathrm{Sq}^{4} Q_{1} \text { and } t \mathrm{Sq}^{2}=t \mathrm{Sq}^{4}=0
$$

implies some $t \theta \neq 0$ where $\theta$ belongs to the algebra generated by elements having $2 \mathrm{Sq}^{1}$ factors. This is impossible by Browder [3]. Hence we get the required contradiction. This completes the inductive proof. The rest of the theorem follows from Lemma 3.5.

\section{General formulas for $Q^{\text {even }}$.}

By analyzing the proof of Theorem 3.6 it should become clear that the Steenrod algebra obeys certain rules that restrict the structure of $Q^{\text {even }}$ as an $a(2)^{+}$module. In this section we generalize Theorem 3.6 to obtain information 
about Steenrod algebra actions on even generators of all degrees. Our starting point is a collection of facts about the Steenrod algebra.

The following ideals of $a(2)$ will appear often in the discussion.

Definition. Let $I_{1}$ be the two-sided ideal of $a(2)$ generated by Sq${ }^{1}$. Let $I_{2}$ be the two-sided ideal generated by $I_{1} \cdot I_{1}$.

Let $J_{r}$ be the right ideal generated by $\mathrm{Sq}^{2}, \mathrm{Sq}^{4}, \ldots, \mathrm{Sq}^{2^{r}}$ for $r \geqq 1$.

Let $K_{r}$ be the right ideal generated by $J_{r}+I_{2}$.

The Adem relations imply the following lemma:

LEMMA 4.1. For $k \geqq 1, r \geqq 1$

(a) $\mathrm{Sq}^{2^{r+1}} k+2^{r} \equiv \mathrm{Sq}^{2^{r}} \bmod K_{r-1}$.

(b) $\mathrm{Sq}^{2 r} \mathrm{Sq}^{2^{r}} \equiv 0 \bmod K_{r-1}$.

(c) $\mathrm{Sq}^{2^{r}} \mathrm{Sq}^{2^{r+1} k} \equiv \lambda \mathrm{Sq}^{r+r+1_{k}} \bmod K_{r-1}, \lambda \in \mathrm{Z}_{2}$.

It will be useful to know when Steenrod operations belong to $K_{r}$ for some $r$.

Lemma 4.2. Suppose $a>0$ is an integer with $a \neq v(s)$ for any $s$. Then if $a$ has dyadic expansion

$$
a=v(j)+2^{i} v(k)+R, \quad i>j+1, k>-1
$$

where $R \equiv 0 \bmod 2^{i+k+2}$ then $\mathrm{Sq}^{a} \in K_{i+k}$.

Proof. The proof is by induction on $a$. If $a=2$ then $\mathrm{Sq}^{2} \in J_{1}$ and $j=-1, k$ $=0, i=1, R=0$. By induction assume the lemma is true for all allowable integers less than $a$.

If $a$ is odd, the Adem relations imply

$$
\mathrm{Sq}^{a}=\mathrm{Sq}^{2^{j+1}} \mathrm{Sq}^{a-2^{j+1}}+\sum_{t=1}^{2^{j}} \alpha_{t} \mathrm{Sq}^{a-t} \mathrm{Sq}^{t}
$$

$a-t$ is an allowable integer. Therefore $\mathrm{Sq}^{a-t} \in K_{i+k}$.

If $a$ is even, then $j=-1$ and if $a \neq 2^{i}$ then

$$
\mathrm{Sq}^{a}=\mathrm{Sq}^{2^{i}} \mathrm{Sq}^{a-2^{i}}+\sum_{t=1}^{2^{i-1}} \alpha_{t} \mathrm{Sq}^{a-t} \mathrm{Sq}^{t}
$$

For even $t, a-t$ is allowable and for odd $t \mathrm{Sq}^{a-t} \mathrm{Sq}^{t} \in I_{2}$. Therefore $\mathrm{Sq}^{a} \in K_{i+k}$ for all allowable $a$.

The following lemmas were used in Theorem 3.6: 
Lemma 4.3. (a) $Q_{j}+\mathrm{Sq}^{v(j)} \in J_{j} \quad$ for $j \geqq 1$.

(b) $\mathrm{Sq}^{v(j)}\left(\mathrm{Sq}^{2^{j+1} k-2^{j}}\right)=0$ for $k \geqq 1$.

Proof. For $j=1, Q_{1}+\mathrm{Sq}^{3}=\mathrm{Sq}^{2} \mathrm{Sq}^{1} \in J_{1}$.

Assume the lemma is true for all integers $j^{\prime}<j$. Then

$$
Q_{j}+\mathrm{Sq}^{v(j)}=\mathrm{Sq}^{2^{j}} Q_{j-1}+\left(Q_{j-1}+\mathrm{Sq}^{v(j-1)}\right) \mathrm{Sq}^{2^{j}} \in J_{j}
$$

by induction. Part (b) follows from the Adem relations.

Definiton. If $K$ is an ideal in $a(2)$, define

$$
\text { Ann } K=\left\{t \in H_{*}\left(X ; \mathbf{Z}_{2}\right) \mid t \theta=0 \text { for } \theta \in K\right\} \text {. }
$$

Lemma 4.4. Let $t \in P H_{2 n}\left(X ; \mathrm{Z}_{2}\right)$. If $t \in \mathrm{Ann} K_{r}, r \geqq 1$ then $(t \otimes t)\left(Q_{r}+\mathrm{Sq}^{v(r)}\right)$ $=0$.

Proof. For $r=1, t \mathrm{Sq}^{2}=0$ implies

$$
(t \otimes t) \mathrm{Sq}^{2} \mathrm{Sq}^{1}=\left(t \mathrm{Sq}^{1} \otimes t \mathrm{Sq}^{1}\right) \mathrm{Sq}^{1}=0 .
$$

Now let $r \geqq 1$ we prove by induction that

$$
(t \otimes t) \mathrm{Sq}^{v(r)}=t \mathrm{Sq}^{v(r)} \otimes t+t \otimes t \mathrm{Sq}^{v(r)} \quad \text { if } t \in \mathrm{Ann} K_{r} .
$$

Suppose the above formula is true for $r^{\prime}<r$. Then $\mathrm{Sq}^{v(r)}=\mathrm{Sq}^{v(r-1)} \mathrm{Sq}^{2^{r}}$ so

$$
\begin{aligned}
(t \otimes t) \mathrm{Sq}^{v(r)} & =\left(t \mathrm{Sq}^{v(r-1)} \otimes t+t \otimes t \mathrm{Sq}^{v(r-1)}\right) \mathrm{Sq}^{2^{r}} \\
& =t \mathrm{Sq}^{v(r)} \otimes t+\sum_{\substack{b \geq 1 \\
a+b=2^{r}}} t \mathrm{Sq}^{v(r-1)} \mathrm{Sq}^{a} \otimes t \mathrm{Sq}^{b}
\end{aligned}
$$

+ symmetric terms .

If $b$ is even, Lemma 4.2 implies $t \mathrm{Sq}^{b}=0$.

If $a$ is odd $\mathrm{Sq}^{v(r-1)} \mathrm{Sq}^{a} \in I_{2}$ so $t \mathrm{Sq}^{v(s-1)} \mathrm{Sq}^{a}=0$.

We conclude

$$
(t \otimes t) \mathrm{Sq}^{v(r)}=t \mathrm{Sq}^{v(r)} \otimes t+t \otimes t \mathrm{Sq}^{v(r)}
$$

Now $t Q_{r}=t \mathrm{Sq}^{v(r)}$ by induction. So $t \otimes t \in \operatorname{kernel}\left(Q_{r}+\mathrm{Sq}^{v(r)}\right)$ if $t \in \operatorname{Ann} K_{r}$.

We can now generalize Theorem 3.6.

Definition. Let

$$
a(s, k)=2^{s+2} k+2 v(s-1)
$$




$$
b(s, k)=2^{s+2} k+2 v(s)
$$

Note that $a(s, k)=b(s-1,2 k)$, and every even integer congruent to two $\bmod 4$ is expressible in the form $a(s, k)$ for unique $s$ and $k$.

THEOREM 4.5. Let $s$, and $k$ be greater than or equal to one. Then

(a) $Q B^{a(s, k)}=\mathrm{Sq}^{2^{s+1} k} Q B^{b(s-1, k)}+\mathrm{Sq}^{2^{s+1}} Q B^{b(s-1,2 k-1)}$.

(b) $\mathrm{Sq}^{2^{s+1}} Q B^{a(s, k)}=0$.

(c) If $t \in P B_{b(s, k)}$ then $t \in$ Ann $K_{s+1}$ or dually $Q B^{b(s, k)} \cap K_{s+1} Q B=0$.

Corollary 4.6. If $\bar{x} \in Q B^{a(s, k)}$ has representative $x$ with $x \neq w^{2}$ and $\bar{\Delta} x \in \xi B \otimes B$, then there are elements $y, z \in B$ with $\bar{\Delta} y \in \xi B \otimes B, \bar{\Delta} z \in \xi B \otimes B$ with

$$
x=\mathrm{Sq}^{2^{s+1} k} y+\mathrm{Sq}^{2^{s+1}} z
$$

and

$$
\mathrm{Sq}^{2^{s+1}} x=0
$$

Proof. This follows from $\left(a_{s}\right)$ and $\left(b_{s}\right)$ and Lemma 3.5, and Theorem 3.3.

Proof of Theorem 4.5. The proof is by induction on $s$. We will prove

(1) $\left(\mathrm{c}_{0}\right)$ implies $\left(\mathrm{a}_{1}\right)$.

(2) $\left(\mathrm{c}_{s-1}\right)$ implies $\left(\mathrm{a}_{s}\right)$.

(3) $\left(c_{s-1}\right)$ and $\left(a_{s}\right)$ imply $\left(b_{s}\right)$.

(4) $\left(\mathrm{c}_{s-1}\right),\left(\mathrm{a}_{s}\right)$ and $\left(\mathrm{b}_{s}\right)$ imply $\left(\mathrm{c}_{s}\right)$.

First note that $\left(\mathrm{c}_{0}\right)$ holds. We must show if $t \in P B_{4 k+2}$ then $t \in \operatorname{Ann} K_{1}$. But $t \mathrm{Sq}^{2} \in P B_{4 k}=0$. Hence $\left(\mathrm{c}_{0}\right)$ is true.

Now $\left(a_{1}\right)$ is precisely Theorem 3.6 , hence $\left(c_{0}\right)$ implies $\left(a_{1}\right)$.

STEP 2. $\left(\mathrm{c}_{s-1}\right)$ implies $\left(\mathrm{a}_{s}\right)$.

We must show $Q B^{a(s, k)}=\mathrm{Sq}^{2^{s+1} k} Q B^{b(s-1, k)}+\mathrm{Sq}^{2^{s+1}} Q B^{b(s-1,2 k-1)}$. Let's consider two preliminary cases.

CASE 1. $\bar{x} \in Q B^{a(s, k)}$ is represented by $x=w^{2}$. Then $w$ has degree $a(s-1, k)+1$. If $k=1$ then

$$
\mathrm{Sq}^{a(s-1, k)+1}=\sum_{l=-1}^{s-1} \mathrm{Sq}^{2 v(s-2)+2^{s+1}-2 v(l)} Q_{l+1} .
$$

Lemma 4.2 implies $\mathrm{Sq}^{2 v(s-2)+2^{s+1}-2 v(l)} \in K_{s+1}$ for all $l$. Therefore $w^{2}$ 
$=\mathrm{Sq}^{a(s-1, k)+1} w$ belongs to $K_{s+1} Q B^{*}$. But $\left(\mathrm{c}_{s-1}\right)$ implies $\overline{w^{2}} \notin K_{s} Q B^{*}$. Therefore $w^{2}=\mathrm{Sq}^{2^{s+1}} \bar{z}$ for some $\bar{z} \in Q B^{b(s-1,2 k-1)}$. If $k>1$ then

$$
\begin{aligned}
\mathrm{Sq}^{a(s-1, k)+1}= & \sum_{l=-1}^{s-3} \mathrm{Sq}^{a(s-1, k)-2 v(l)} Q_{l+1}+\mathrm{Sq}^{2^{s+1} k} Q_{s-1}+ \\
& +\left(Q_{s}+\mathrm{Sq}^{v(s)}\right) \mathrm{Sq}^{2^{s+1} k-2^{s}}
\end{aligned}
$$

By Lemma 4.3(b), $Q_{s}+\mathrm{Sq}^{v(s)} \in J_{s}$ and $\mathrm{Sq}^{a(s-1, k)-2 v(l)} \in K_{s-1}$ by Lemma 4.2. Therefore $c_{s-1}$ implies $\bar{w}^{2} \in \mathrm{Sq}^{2^{s+1} k} Q B^{b(s-1, k)}$. This completes Case 1 .

CASE 2. $\bar{x} \in Q B^{a(s, 1)}, x \neq w^{2}$. By Theorem $2.3 \bar{x} \in \operatorname{Ia}(2)^{+} Q B$. But $\left(\mathrm{c}_{s-1}\right)$ implies $\bar{x} \notin K_{s} Q B$. Since $a(2)^{+}$is generated by $\mathrm{Sq}^{2^{i}}, \bar{x}=\mathrm{Sq}^{2^{i}} \bar{y}$ for some $i$. For degree reasons we must have $i=s+1$. This completes Case 2 .

We may now assume

CASE 3. $\bar{x} \in Q B^{a(s, k)}, k>1$ and $x \neq w^{2}$. By induction we may assume the theorem holds for all $Q B^{a\left(s, k^{\prime}\right)}$ where $k^{\prime}>k$.

Choose $x$ so that $\bar{\Delta} x \in \xi B \otimes B$ and $x \in B(m+1)$, and suppose

$$
x \notin B(m)+\mathrm{im} \mathrm{Sq}^{2^{s+1} k}+\mathrm{im} \mathrm{Sq}{ }^{2^{s+1}} .
$$

We will show this leads to a contradiction. There is a primitive $t \in P B_{a(s, k)}$ with $\langle t, x\rangle \neq 0$ and

$$
\left\langle t, B(m)+\mathrm{im} \mathrm{Sq}^{2^{s+1} k}+\mathrm{im} \mathrm{Sq}^{2^{s+1}}\right\rangle=0 .
$$

$\left(\mathrm{c}_{s-1}\right)$ and (4.1.1) imply

$$
t \in \operatorname{Ann} K_{s+1} .
$$

Consider the factorization

$$
\begin{aligned}
\mathrm{Sq}^{1} \mathrm{Sq}^{a(s, k)}= & \sum_{l=-1}^{s-2} \mathrm{Sq}^{2^{s+2} k+2 v(s-1)-2 v(l)} Q_{l+1}+\mathrm{Sq}^{2^{s+2} k} Q_{s}+ \\
& +\left(Q_{s+1}+\mathrm{Sq}^{v(s+1)}\right) \mathrm{Sq}^{2^{s+2} k-2^{s+1}}
\end{aligned}
$$

Since $k>1$, Lemma 4.1 (c) implies

$$
\mathrm{Sq}^{2^{s+2} k-2^{s+1}} \equiv \mathrm{Sq}^{2^{s+1}} \mathrm{Sq}^{2^{s+2}(k-1)} \bmod K_{s} .
$$

$\mathrm{Sq}^{2^{s+2}(k-1)} x \in B^{a(s, 2 k-1)}$ where $2 k-1>k$ because $k>1$. By induction there are generators $\bar{x}_{1}, \bar{x}_{2} \in Q B$ with

$$
\mathrm{Sq}^{2^{s+2}(k-1)} \bar{x}=\mathrm{Sq}^{2^{s+1}(2 k-1)} \bar{x}_{1}+\mathrm{Sq}^{2^{s+1}} \bar{x}_{2} .
$$

If $x_{1}$ and $x_{2}$ are chosen so that $\bar{\Delta} x_{i} \in \xi B \otimes B$ then by Lemma 3.5 we may assume 


$$
\mathrm{Sq}^{2^{s+2}(k-1)} x=\mathrm{Sq}^{2^{s+1}(2 k-1)} x_{1}+\mathrm{Sq}^{2^{s+1}} x_{2}
$$

Hence

$$
\begin{aligned}
\mathrm{Sq}^{2^{s+1}} \mathrm{Sq}^{2^{s+1}(k-1)} x & =\mathrm{Sq}^{2^{s+1}} \mathrm{Sq}^{2^{s+1}(2 k-1)} x_{1}+\mathrm{Sq}^{2^{s+1}} \mathrm{Sq}^{2^{s+1}} x_{2} \\
& =0
\end{aligned}
$$

by Lemma 3.5 and $\left(c_{s-1}\right)$.

Equation (4.1.4), $\mathrm{c}_{s-1}$ and Lemma 3.5 imply

$$
\mathrm{Sq}^{2^{s+2} k-2^{s+1}} x=0 \text {. }
$$

We also have $Q_{i} x=0$ because $x \in B$. Hence Main Theorem 1.3 of [8] applies. If we show

(4.1.6) $t \otimes t \in \operatorname{kernel}\left[\sum \mathrm{Sq}^{2^{s+2} k+2 v(s-1)-2 v(l)}+\mathrm{Sq}^{2^{s+2} k}+\left(Q_{s+1}+\mathrm{Sq}^{v(s+1)}\right)\right]$

this would imply $t^{2} \neq 0$ which is a contradiction. We proceed to prove (4.1.6).

It will be convenient to use the notation

$$
t \mathrm{Sq}^{a \pm u}=t \mathrm{Sq}^{a-u} \otimes t \mathrm{Sq}^{a+u}+t \mathrm{Sq}^{a+u} \otimes t \mathrm{Sq}^{a-u}
$$

Carefully checking degrees, we get

$$
(t \otimes t) \mathrm{Sq}^{2^{s+2} k+2 v(s-1)-2 v(l)}=\sum_{0<u<v(l)} t \mathrm{Sq}^{2^{s+1} k+v(s-1)-v(l) \pm u} .
$$

Here we use the property $t \mathrm{Sq}^{r}=0$ if $r \geqq \frac{1}{2}$ degree $t$. But by Lemma 4.2, $\mathrm{Sq}^{2^{s+1} k+v(s-1)-v(l)+u} \in K_{s-1}$; therefore,

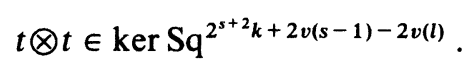

Now consider

$$
(t \otimes t) \mathrm{Sq}^{2^{s+2} k}=\sum_{0<u<v(s-1)} t \mathrm{Sq}^{2^{s+1} k \pm u} .
$$

If $u=0, t \mathrm{Sq}^{2^{s+1} k}=0$ by construction. If $u \neq v(j)$ for some $j<(s-1)$ then Lemma 4.2 implies

$$
\mathrm{Sq}^{2^{s+1} k \pm u} \in K_{s-1} .
$$

If $u=v(j)$ there is a factorization

$$
\begin{aligned}
\mathrm{Sq}^{2^{s+1} k+v(j)}= & \sum_{l=-1}^{j-1} \mathrm{Sq}^{2^{s+1} k+2 v(j-1)-2 v(l)} Q_{l+1}+ \\
& +\left(Q_{j+1}+\mathrm{Sq}^{v(j+1)}\right) \mathrm{Sq}^{2^{s+1} k-2^{j+1}}
\end{aligned}
$$

Lemma 4.2, Lemma 4.3 and (4.1.8) imply

$$
\mathrm{Sq}^{2^{s+2} k+v(j)} \in K_{s-1} \text {. }
$$


Hence $t \otimes t \in \operatorname{ker~Sq}{ }^{2^{s+2} k}$.

Finally Lemma 4.4 together with (4.1.2) imply

$$
t \otimes t \in \operatorname{kernel}\left(Q_{s+1}+\mathrm{Sq}^{v(s+1)}\right) .
$$

By Main Theorem 1.3 we get $t^{2} \neq 0$ which is a contradiction. This proves Case 3.

STEP 3. $\left(\mathrm{c}_{s-1}\right)$ and $\left(\mathrm{a}_{s}\right)$ imply $\left(\mathrm{b}_{s}\right)$.

To show $\mathrm{Sq}^{2^{s+1}} Q B^{a(s, k)}=0$, note $\mathrm{Sq}^{2^{s+1}} Q B^{a(s, k)} \cong Q B^{b(s-1,2 k+1)}$ and $\left(\mathrm{c}_{s-1}\right)$ implies

$$
Q^{b(s-1,2 k+1)} \cap K_{s} Q B^{*}=0 .
$$

But $\left(\mathrm{a}_{s}\right)$ implies

$$
Q B^{a(s, k)}=\mathrm{Sq}^{2^{s+1} k} Q B^{b(s-1, k)}+\mathrm{Sq}^{2^{s+1}} Q B^{b(s-1,2 k-1)} .
$$

Lemma 4.1 implies

$$
\mathrm{Sq}^{2^{s+1}} Q B^{a(s, k)} \cong \lambda \mathrm{Sq}^{2^{s+1}(k+1)} Q B^{b(s-1, k)}=0 .
$$

This completes the proof of Step 3.

STEP 4. $\left(\mathrm{c}_{s-1}\right)$, $\left(\mathrm{a}_{s}\right)$, and $\left(\mathrm{b}_{s}\right)$ imply $\left(\mathrm{c}_{s}\right)$.

To prove if $t \in P B_{b(s, k)}$ then $t \in \operatorname{Ann} K_{s+1}$. By $\left(\mathrm{c}_{s-1}\right) t \in \operatorname{Ann} K_{s}$. It therefore suffices to prove $t \mathrm{Sq}^{2^{s+1}}=0$.

But if $t \mathrm{Sq}^{2^{s+1}} \neq 0$ that would imply $\mathrm{Sq}^{2^{s+1}} Q B^{a(s, k)} \neq 0$ which contradicts $\left(\mathrm{b}_{s}\right)$. This proves Step 4.

Steps 1-4 prove the theorem by induction on $s$.

\section{REFERENCES}

1. P. Baum and W. Browder, The cohomology of quotients of classical groups, Topology 3 (1965), 305-336.

2. R. Bott, On torsion in Lie groups, Proc. Nat. Acad. Sci. U.S.A. 40 (1954), 586-588.

3. W. Browder, Torsion in H-spaces, Ann. of Math. 74 (1961), 24-51.

4. J. R. Hubbuck and R. Kane, On $\pi_{3}$ of a finite H-space, Trans. Amer. Math. Soc. 213 (1975), 99-105.

5. R. Kane, The module of indecomposables for finite H-spaces I, II, Trans. Amer. Math. Soc. 222, (1976), 303-318. II to appear.

6. R. Kane, On loop spaces without p-torsion, Pacific J. Math. 60 (1975), 189-201.

7. J. Lin, Steenrod squares in the mod 2 cohomology of a finite H-space, Comment. Math. Helv. (to appear). 
8. J. Lin, Torsion in H-spaces I, II, Ann. of Math. 103 (1976), 457-487, 107 (1978), 41-88.

9. J. Milnor, The Steenrod algebra and its dual, Ann. of Math. 67 (1958), 150-171.

10. J. Milnor and J. C. Moore, On the structure of Hopf algebras, Ann. of Math. 81 (1965), 211264.

11. N. E. Steenrod and D. B. A. Epstein, Cohomology Operations, Annals of Math. Studies 50, Princeton University Press, Princeton, 1962.

DEPARTMENT OF MATHEMATICS

UNIVERSITY OF CALIFORNIA, SAN DIEGO

LA JOLLA, CALIFORNIA 92093, U.S.A. 